\title{
A SIMULATION SURVEY OF GALAXY INTERACTIONS
}

\author{
G. Byrd, W. Keel(U. of Alabama) and S. Howard(Los Alamos)
}

\section{Introduction}

Many carefully selected samples of interacting galaxies have been observed extensively in attempts to clarify whether interaction produces activity in galaxies. Because the sample members represent a wide range of encounter parameters and times, one can then study whether there are correlations between observable encounter features and, for example, Seyfert activity. On the other hand, in theoretical studies, simulations typically deal with either time-consuming detailed modelling of single galaxy pairs or tracing a few model encounters over time. We are extending the observational survey approach by combining it with a simulation survey.

\section{Simulations}

We are conducting a survey of model encounters, covering the most important encounter parameters over a wide range. Some parameters, such as companion structure and initial velocity, are demonstratably less important and can be ignored in a first pass. The parameter range must be richly enough sampled so that we can evaluate the uniqueness of the observable morphology and velocity structure of the resulting simulated pairs to diagnose unobservable companion orbit parameters. We are using use a self-gravitating polar n-body code run on the Cray X-MP at the Alabama Supercomputer Network.

\section{Comparison with Observational Samples}

For each simulation, we will have stellar and gas distributions predicted over, typically, a billion years, along with information on gas motions within the disk and any material captured by the companion or lost to the system. Features of disturbed spiral galaxies are sensitive enough to time and encounter parameters so that a match of the simulation survey results to observations can be applied as starting points to infer unobservable orbital or system parameters in actual sample members. This should enable us to examine whether interesting observed properties (Seyfert activity, nuclear star-formation rate) are functions of unobservable dynamical properties which characterize each encounter. Any correlations (or lack of some expected ones!) will provide strong clues as to how or whether these phenomena are related to interactions. Aside from its use with such observed samples, this survey should greatly speed determination of initial orbital parameters for more detailed subsequent simulations of individual systems. 


\section{Display and Publication}

The images are in IRAF format with $256 \times 256$ cells. Publication of a portion of the images via normal hardcopy is planned. However, the entire survey will be available on tape in FITS format so comparison with observations can be made on computer image processing systems.

\section{Disk Parameters and Model Approximations}

The self-gravitating disk has a finite radius with initially constant rotation curve (Mestel disk). In the disk, 30 percent of the particles are "gas" with no velocity dispersion and the rest "stars" with the appropriate stabilizing dispersion. The simulation code is a FFT particle-mesh code with a polar grid with bin size proportional to radius (small near center, large near edge as needed for disk simulations). Experience shows a 24 radial by 36 azimuthal grid with 180,000 particles provides reasonable spatial resolution and sufficient numbers of particles per bin. The disk initially fills about one-third of the grid.

To maximize disk particle number, we make the following approximations: a 2 D self-gravitating disk, inert halo, and softened inert companion. These are reasonable for most encounters, but more elaborate simulations will be made where necessary. Because of uncertainty, we use halo-to-disk ratios of 1.0 and 10.0.

\section{Encounter Parameters (Kicking the Disk in Different Ways)}

We survey a variety of encounter parameter values. Companion orbit inclination values are $0,30,60$, and 89 degrees. Orbit sense direct and retrograde relative to the disk "spin" will be tried. Initial encounter distances of one(grazing) and two times the disk radius will be simulated. Companion masses of $0.1,0.5$, and 1.0 times the target galaxy will be assumed. Initial encounter orbits will be assumed to be parabolic.

\section{Examples of Results}

Fig. 1 shows a set of 0 degree inclination, grazing encounters. The target galaxy turns counter clockwise. Direct companions orbit counter clockwise, retrograde clockwise. About 750 out of 1000 steps are shown with 50 steps between frames. A disk orbital period is about 300 steps. The left box is a direct encounter; the right, retrograde. Both have a 0.1 galaxy mass companion.

\section{Example of Comparison with Observation}

Fig. 2 shows photographs of systems and inset frames from our initial survey results. The left photo (NGC 2535/6) matches one of the direct Fig. 1 small-mass encounter shapes. The right photo (Arp 107) matches a direct 0.5 galaxy-mass companion encounter.(La Palma photos) We emphasize that the simulation frames were simply picked from the survey, showing how good fits to disk morphology can be easily obtained. 


\section{Some Encounter Morphology Generalizations}

Direct encounters seem to preferentially generate the usual two-armed patterns with some interesting bends and kinks in the arms. See Fig. 1 left. Retrograde encounters are generally less violent in their effects but they can create interesting "scallop" patterns, off-center rings, and leading arms in the inner portions for strongly perturbed disks. The second from top right frame in the Fig. 1 retro encounter shows a scallop pattern. Such details are much clearer on an image processing system. Inclined encounters tend to generate one-sided patterns opposite to those seen in disk-plane encounters.

\section{ACKNOWLEDGEMENTS}

This work was supported in part by NSF/EPSCoR grant RII 8610669 and by Cray Research Inc. grant UA6. Computer time is provided by the Alabama Supercomputer Network. Our much-modified and extensively rewritten code is based on one kindly provided by R. Miller, who pioneered polar n-body code development. Photgraphs are from the Jacobus Kapteyn Telescope (operated by the Royal Greenwich Observatory at the Observatorio del Rogue de los Muchachos on La Palma).

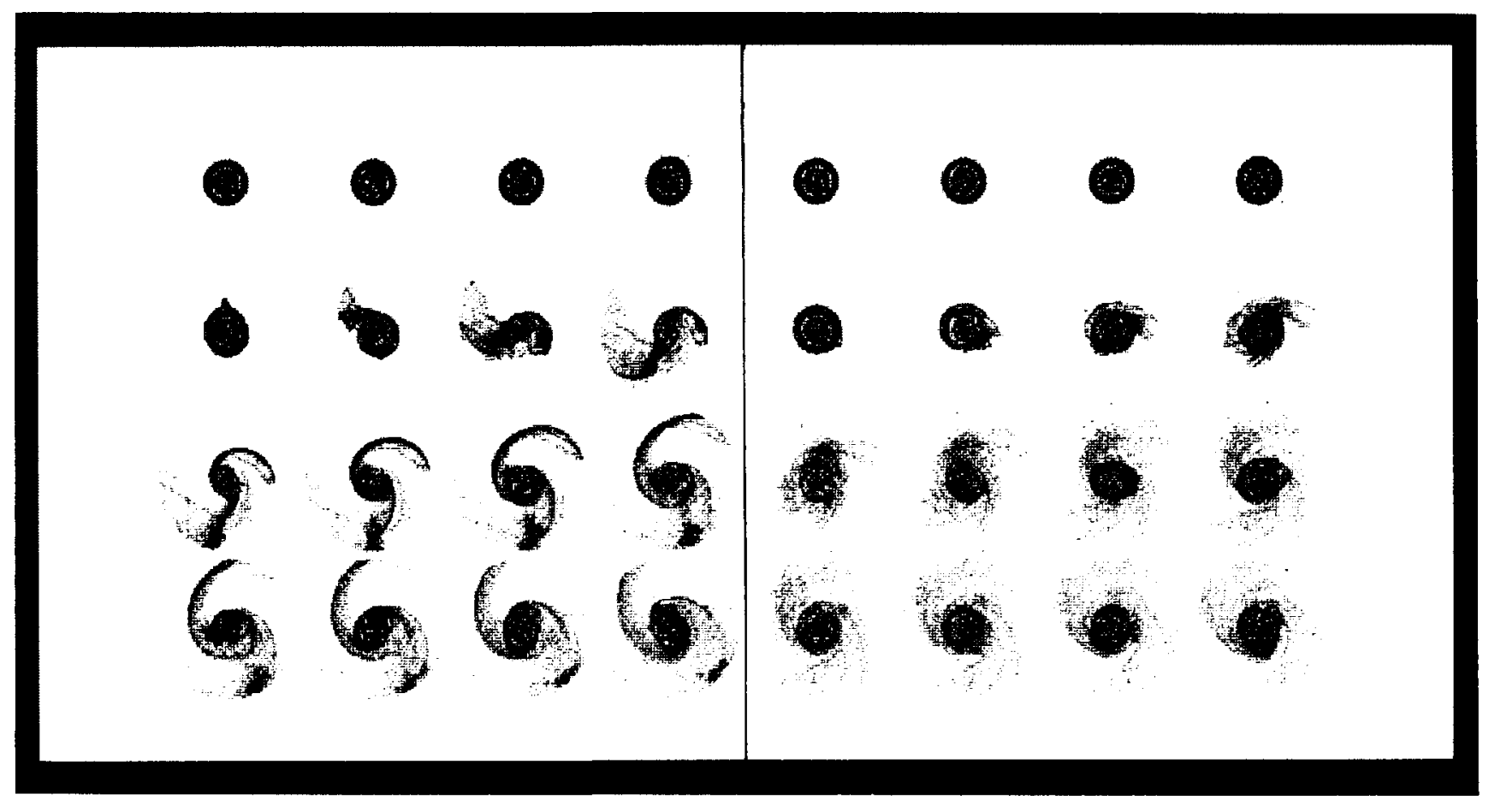

Fig. 1. Direct(1), Retrograde(r), "Gas," Companion/Galaxy 0.1 


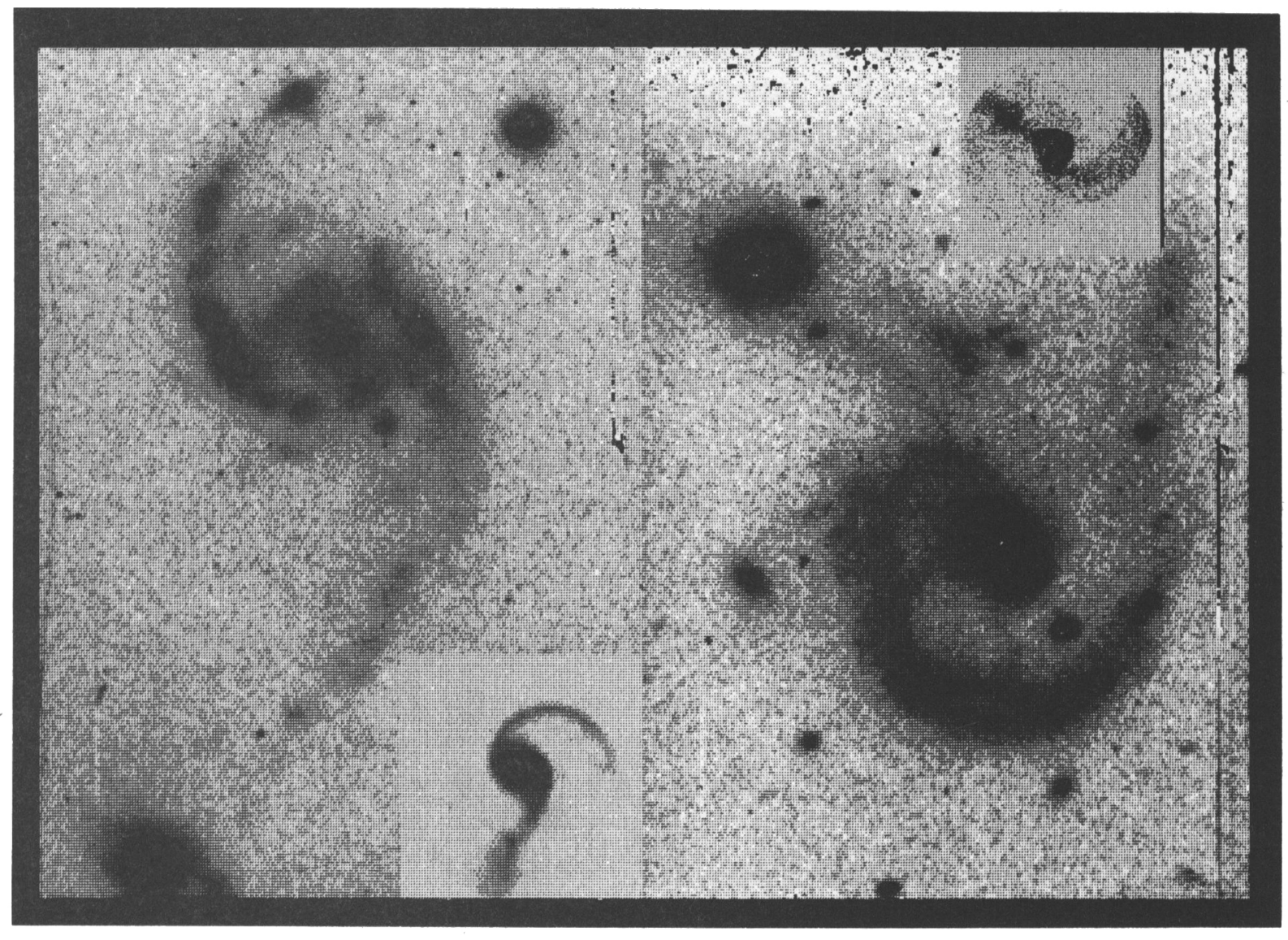

Fig. 2. Examples of matching survey frames to observations. 